\title{
A POINTWISE LIPSCHITZ SELECTION THEOREM
}

\author{
MIEK MESSERSCHMIDT
}

\begin{abstract}
We prove that any correspondence (multi-function) mapping a metric space into a Banach space that satisfies a certain pointwise Lipschitz condition, always has a continuous selection that is pointwise Lipschitz on a dense set of its domain.

We apply our selection theorem to demonstrate a slight improvement to a well-known version of the classical Bartle-Graves Theorem: Any continuous linear surjection between infinite dimensional Banach spaces has a positively homogeneous continuous right inverse that is pointwise Lipschitz on a dense meager set of its domain.

An example devised by Aharoni and Lindenstrauss shows that our pointwise Lipschitz selection theorem is in some sense optimal: It is impossible to improve our pointwise Lipschitz selection theorem to one that yields a selection that is pointwise Lipschitz on the whole of its domain in general.
\end{abstract}

\section{INTRODUCTION}

Many correspondences (multi-functions) exhibit some form of Lipschitz behavior. A classical example is what may be termed the inverse image correspondence of a continuous linear surjection between Banach spaces: Let $X$ and $Y$ be Banach spaces and $T: X \rightarrow Y$ a continuous linear surjection. We define the inverse image correspondence $\varphi: Y \rightarrow 2^{X}$ by $\varphi(y):=T^{-1}\{y\}$ for all $y \in Y$. It is easily seen that a map $\tau: Y \rightarrow X$ is a selection of $\varphi$ (meaning $\tau(y) \in \varphi(y)$ for all $y \in Y$ ) if and only if $\tau$ is a right inverse of $T$. It is well-known, by the Bartle-Graves Theorem (a version is stated as Theorem 5.1 in this paper), that there always exists a continuous selection of $\varphi$. Modern proofs of this version of the Bartle-Graves Theorem, e.g. [2, Corollary 17.67], proceed through a straightforward application of Michael's Selection Theorem (stated in this paper as Theorem 2.1).

By the Open Mapping Theorem, it can be seen that the correspondence $\varphi$ is Lipschitz when $2^{X}$ is endowed with the Hausdorff distance. Furthermore, the correspondence $\varphi$ also exhibits a form of pointwise Lipschitz behaviour. Again, by the Open Mapping Theorem, it can be seen that there exists a constant $\alpha \geq 0$ so that, for any $y \in Y$ and $x \in \varphi(y)$, the correspondence $\psi: Y \rightarrow 2^{X}$, defined by $\psi(z):=\varphi(z) \cap\left(x+\alpha\|y-z\| \mathbf{B}_{X}\right)$ for all $z \in Y$, is non-empty-valued. All selections $\tau: Y \rightarrow X$ of $\psi$ (continuous or not) will therefore be strongly pointwise $\alpha$-Lipschitz at $y$ (by which we mean $\|\tau(y)-\tau(z)\| \leq \alpha\|y-z\|$ for all $z \in Y$ ).

Even though the correspondence $\varphi$ always exhibits some form of Lipschitz behavior, an example devised by Aharoni and Lindenstrauss (cf. 11] and [3, Example 1.20]) shows that it is however impossible establish the existence of Lipschitz selections of inverse image correspondences in general. Godefroy and Kalton gave a characterization of the continuous linear surjections between separable Banach spaces admitting Lipschitz right inverses as exactly the ones with continuous linear right inverses, and hence, as exactly those with complemented kernels (cf. 9, Corollary 3.2]). However, this does not extend to non-separable Banach spaces (cf.

2010 Mathematics Subject Classification. 54C65, 54C60 (primary), and 46B99 (secondary). Key words and phrases. Selection theorem, pointwise Lipschitz map, Bartle-Graves Theorem. The author's research was funded by The Claude Leon Foundation. 
[10, Section 2.2]). We also refer the reader to the negative result [12, Theorem 2.4] by Przesławski and Yost.

Still, the Lipschitz-like behaviour of the inverse image correspondence of a continuous linear surjection between Banach spaces (and other related correspondencesin) raises the following question:

Question. Given the general Lipschitz-like behavior of the inverse image correspondence $\varphi$, as defined above, do there exist selections of $\varphi$ that exhibit more regularity than the mere continuity ensured by the classical Bartle-Graves Theorem? More generally, is there a theorem for correspondences displaying such Lipschitz-like behavior, akin to Michael's Selection Theorem, asserting the existence of selections which exhibit more regularity than mere continuity?

We will give one positive answer to this question in this paper. Our main goal in this paper is to prove a general Pointwise Lipschitz Selection Theorem (Theorem 3.4). This result gives sufficient conditions under which a correspondence always admits a continuous selection that is pointwise Lipschitz on a dense set of its domain. Explicitly:

Theorem 3.4 (Pointwise Lipschitz Selection Theorem). Let $(M, d)$ be a metric space and $X$ a Banach space. Let $\alpha \geq 0$ and let $\varphi: M \rightarrow 2^{X}$ be a non-empty-, closed-, and convex-valued correspondence that admits local strongly pointwise $\alpha$ Lipschitz selections (as defined in Definition 3.1). If $\varphi$ has a (bounded) continuous selection, then, for every $\beta>\alpha$, there exists a (bounded) continuous selection of $\varphi$ that is pointwise $\beta$-Lipschitz (as defined in Definition [2.6) on a dense set of $M$.

The proof proceeds through a somewhat delicate inductive construction which is performed in proving Lemma [3.2 There we prove the existence of a uniform Cauchy sequence of continuous selections that are pointwise Lipschitz on the points successively larger maximal separations of $M$ (cf. Definition 2.3). Each selection in this sequence is constructed as a slight adjustment of its predecessor so as to be pointwise Lipschitz at more points. This is done while also taking care that our adjustments do not disturb the predecessor where it is already known to be pointwise Lipschitz. The limit of this sequence is then shown to have the desired properties in the proof of Theorem 3.4. We refer the reader to Section 3 for further details.

The condition of admitting local strongly pointwise $\alpha$-Lipschitz selections required in the hypothesis of the above theorem is admittedly somewhat synthetic. The reason for working with this condition in favor of a more natural, more easily verified condition, is purely to abstract out the important features required in the proof of Theorem 3.4. Yet, the critical reader may well raise the question: Why should correspondences with this property even exist? In reply, we introduce the more natural notion of "lower pointwise Lipschitz-ness" of a correspondence (cf. Definition 4.1). Section 4 is devoted to showing that being lower pointwise Lipschitz is sufficient for a correspondence to admit strongly pointwise Lipschitz selections. This allows us to prove versions of Theorem 3.4 in Corollaries 4.5 and 4.6 which are slightly less general, yet slightly more natural.

Corollary 4.5. Let $(M, d)$ be a metric space, $X$ a Banach space and $\alpha \geq 0$. Let $\varphi: M \rightarrow X$ be a closed-and convex-valued lower hemicontinuous correspondence that is lower pointwise $(\alpha+\varepsilon)$-Lipschitz for every $\varepsilon>0$. Then, for any $\beta>\alpha$, there exists a continuous selection of $\varphi$ that is pointwise $\beta$-Lipschitz on a dense set of $M$.

\footnotetext{
${ }^{1}$ For example, for Banach spaces $X, Y$ and a closed cone $C \subseteq X$, consider the inverse image correspondence of a continuous additive positively homogeneous surjection $T: C \rightarrow Y$ (cf. [5]).
} 
If, additionally, there exists a bounded continuous selection of $\varphi$, then, for any $\beta>\alpha$, there also exists a bounded continuous selection of $\varphi$ that is pointwise $\beta$ Lipschitz on a dense set of $M$.

Corollary 4.6. Let $(M, d)$ be a bounded metric space, $X$ a Banach space and $\alpha \geq 0$. Let $\varphi: M \rightarrow X$ be a closed-and convex-valued lower hemicontinuous correspondence that is lower pointwise $(\alpha+\varepsilon)$-Lipschitz for every $\varepsilon>0$. Then, for any $\beta>\alpha$, there exists a bounded continuous selection of $\varphi$ that is pointwise $\beta$-Lipschitz on a dense set of $M$.

As an illustrative application, we establish the following slightly improved version of the classical Bartle-Graves Theorem:

Theorem 5.2 (Improved Bartle-Graves Theorem). Let $X$ and $Y$ be infinite dimensional Banach spaces and $T: X \rightarrow Y$ a continuous linear surjection. Then there exists a constant $\eta>0$ and a positively homogeneous continuous right inverse $\tau: Y \rightarrow X$ of $T$ that is pointwise $\eta$-Lipschitz on a dense meager set of $Y$.

We note that Theorem 3.4 is, in some sense, optimal. To elaborate, in general it is impossible to conclude that a selection yielded by Theorem 3.4 is pointwise Lipschitz on its entire domain $M$. Should this be the case in general, a result adapted from Durand-Cartagena and Jaramillo [8, Corollary 2.4] in combination with a result by Schäffer [13, Theorem 3.5]2, will show that an inverse image correspondence will always admit a Lipschitz selection. This however contradicts the above mentioned example devised by Aharoni and Lindenstrauss of an inverse image correspondence that admits no Lipschitz selection. We refer the reader to Section 6 for further details.

We give a brief outline of the organization of the paper.

In Section 2, we provide the notation and definitions used throughout this paper. Some elementary preliminary results are also proven. Specifically, Section 2.4 gives some quite elementary results on so-called separations in metric spaces, and Section 2.5 proves some basic results on pointwise Lipschitz functions.

Section 3 will establish our main result, Theorem 3.4. The proof of this theorem is presented in two steps. Firstly, we give sufficient conditions for a correspondence to have a uniform Cauchy sequence of continuous selections, where the members of this sequence are pointwise Lipschitz on successively finer separations (cf. Lemma 3.2). The second step analyses the limit of such a Cauchy sequence of selections and shows the limit is a selection which is pointwise Lipschitz on a dense set of its domain (cf. Theorem 3.4).

In Section 4 we define the notion of lower pointwise Lipschitz-ness of a correspondence (cf. Definition 4.1). This property is more natural than that of admitting local strongly pointwise Lipschitz selections as required in the hypothesis of Theorem 3.4 Proposition 4.4 shows that lower pointwise Lipschitz-ness of a correspondence is sufficient for Theorem 3.4 to be applicable to the correspondence, and results in the somewhat more natural versions of Theorem 3.4 in Corollaries 4.5 and 4.6 .

We give an application of our Pointwise Lipschitz Selection Theorem in Section 5 by establishing a slightly improved version (Theorem 5.2) of the classical BartleGraves Theorem.

Finally, in Section [6 we briefly discuss the significance of an example devised by Aharoni and Lindenstrauss to our results. Specifically, we argue why Theorem 3.4

\footnotetext{
${ }^{2}$ Proven independently by the author and Wortel [11, Theorem 3.6], while in ignorance of Schäffer's work.
} 
is, in some sense, the best possible general Lipschitz selection theorem one can hope to prove.

\section{Preliminary Definitions, Results AND NOtation}

2.1. Notation for balls in metric spaces. For a metric space $(M, d)$ with $a \in$ $M$ and $r>0$, we will denote the open and closed balls with radius $r$ about $a$ respectively by

$$
\begin{aligned}
\mathbb{B}_{M}(a, r) & :=\{b \in M \mid d(a, b)<r\}, \\
\mathbf{B}_{M}(a, r) & :=\{b \in M \mid d(a, b) \leq r\} .
\end{aligned}
$$

Let $X$ be a normed space. We denote the open unit ball, closed unit ball and unit sphere respectively by $\mathbb{B}_{X}, \mathbf{B}_{X}$ and $\mathbf{S}_{X}$. To aid in readability by reducing nested parentheses, for $x \in X$ and $r>0$, we will sometimes write $x+r \mathbb{B}_{X}$ and $x+r \mathbf{B}_{X}$ instead of $\mathbb{B}_{X}(x, r)$ and $\mathbf{B}_{X}(x, r)$. We will view any subset of $X$ as a metric space with the metric induced from the norm on $X$.

2.2. Spaces of functions. Let $F$ be a Hausdorff space and $X$ a normed space. By $C(F, X)$ we will denote the normed space of all bounded continuous functions on $F$ taking values in $X$, endowed with the uniform norm $\|\cdot\|_{\infty}$. A standard argument shows that $C(F, X)$ is a Banach space whenever $X$ is a Banach space. For any function $f: F \rightarrow X$ and $G \subseteq F$, we denote the restriction of $f$ to $G$ by $\left.f\right|_{G}: G \rightarrow X$.

2.3. Correspondences. Let $A, B$ be sets. By a correspondence we mean a setvalued map $\varphi: A \rightarrow 2^{B}$ and we will use the notation $\varphi: A \rightarrow B$. We will say $\varphi$ is non-empty-valued (respectively, convex-valued or closed-valued) if $\varphi(a)$ is nonempty (respectively, convex or closed) for all $a \in A$ (granted that these notions make sense in $B$ ).

Let $A$ and $B$ be topological spaces and $\varphi: A \rightarrow B$ be any correspondence. We will say that $\varphi$ is lower hemicontinuous at $a \in A$, if, for every open set $U \subseteq B$ satisfying $\varphi(a) \cap U \neq \emptyset$, there exists some open set $V \ni a$ satisfying $\varphi(v) \cap U \neq \emptyset$ for all $v \in V$. We will say that $\varphi$ is lower hemicontinuous, if $\varphi$ is lower hemicontinuous at every point in $A$. By a selection of $\varphi$ we mean a function $f: A \rightarrow B$ satisfying $f(a) \in \varphi(a)$ for all $a \in A$.

We quote the following two well-known classical results that we will need in later sections.

Theorem 2.1 (Michael's Selection Theorem [2, Theorem 17.66]). Let $P$ be a paracompact space and $X$ a Banach space. Every non-empty-, closed-and convexvalued lower hemicontinuous correspondence $\varphi: P \rightarrow X$ has a continuous selection.

Theorem 2.2 (Stone's Theorem [14, Corollary 1]). Every metric space is paracompact.

2.4. Separations in metric spaces. In this section we give some basic definitions and results concerning separations in metric spaces. Lemmas 2.4 and 2.5] are elementary verifications whose proofs we omit.

Definition 2.3. Let $(M, d)$ be a metric space. For $r>0$, a set $B \subseteq M$ will be called an $r$-separation in $M$, if, for distinct $a, b \in B$, we have $d(a, b) \geq r$. We partially order the set of all $r$-separations in $M$ by inclusion.

A straightforward application of Zorn's Lemma will establish:

Lemma 2.4. Let $(M, d)$ be a metric space. Let $r>0$ and let $B$ be an $r$-separation in $M$. Then there exists a maximal $r$-separation in $M$ containing $B$. 
Lemma 2.5. Let $(M, d)$ be a metric space and let $\left\{r_{n}\right\}$ be any descending sequence of positive real numbers that converges to zero. For each $n \in \mathbb{N}$, let $B_{n}$ be a maximal $r_{n}$-separation with $B_{n-1} \subseteq B_{n}$ (where we set $B_{0}:=\emptyset$ ). Then $\bigcup_{n \in \mathbb{N}} B_{n}$ is dense in $M$.

2.5. Pointwise Lipschitz maps. In this section we introduce the notion of pointwise Lipschitz functions.

Definition 2.6. Let $(M, d)$ and $\left(M^{\prime}, d^{\prime}\right)$ be metric spaces, $\alpha \geq 0$ and $f: M \rightarrow M^{\prime}$ any map.

(1) We will say $f$ is pointwise $\alpha$-Lipschitz at $b \in M$, if

$$
\limsup _{r \rightarrow 0^{+}}\left(r^{-1} \sup \left\{d^{\prime}(f(b), f(a)) \mid a \in \mathbf{B}_{M}(b, r)\right\}\right) \leq \alpha .
$$

For a set $S \subseteq M$, we will say $f$ is pointwise $\alpha$-Lipschitz on $S$ if $f$ is pointwise $\alpha$-Lipschitz at every point of $S$.

(2) We will say $f$ is strongly pointwise $\alpha$-Lipschitz S $^{3} b \in M$, if, for all $a \in M$,

$$
d^{\prime}(f(b), f(a)) \leq \alpha d(b, a) .
$$

The following somewhat technical lemmas will be needed in later sections. In summary, Lemma 2.7 shows that we may replace closed balls with open balls in the definition of pointwise Lipschitz-ness, and Lemma 2.8 shows that pointwise Lipschitz-ness is preserved by homogeneous extensions of bounded functions.

Lemma 2.7. Let $(M, d)$ and $\left(M^{\prime}, d^{\prime}\right)$ be metric spaces and $\alpha \geq 0$. For some $b \in M$, a function $f: M \rightarrow M^{\prime}$ is pointwise $\alpha$-Lipschitz at $b$ if and only if

$$
\limsup _{r \rightarrow 0^{+}}\left(r^{-1} \sup \left\{d(f(b), f(a)) \mid a \in \mathbb{B}_{M}(b, r)\right\}\right) \leq \alpha .
$$

Proof. Let $f$ be pointwise $\alpha$-Lipschitz at $b$. Since $\mathbb{B}_{M}(b, r) \subseteq \mathbf{B}_{M}(b, r)$ for all $r>0$, we have

$$
\begin{aligned}
\limsup _{r \rightarrow 0^{+}} & \left(r^{-1} \sup \left\{d(f(b), f(a)) \mid a \in \mathbb{B}_{M}(b, r)\right\}\right) \\
& \leq \limsup _{r \rightarrow 0^{+}}\left(r^{-1} \sup \left\{d(f(b), f(a)) \mid a \in \mathbf{B}_{M}(b, r)\right\}\right) \\
& \leq \alpha .
\end{aligned}
$$

Conversely, let $\limsup _{r \rightarrow 0^{+}}\left(r^{-1} \sup \left\{d(f(b), f(a)) \mid a \in \mathbb{B}_{M}(b, r)\right\}\right) \leq \alpha$. Let $\varepsilon>$ 0 be arbitrary. Then there exists some $s>0$ such that, for all $r \in(0, s)$, we have

$$
r^{-1} \sup \left\{d(f(b), f(a)) \mid a \in \mathbb{B}_{M}(b, r)\right\}<\alpha+2^{-1} \varepsilon .
$$

Let $r \in(0, s)$ be arbitrary. Then, for any $\kappa>0$ satisfying

$$
\kappa<\min \left\{2^{-1} r \varepsilon\left(\alpha+2^{-1} \varepsilon\right)^{-1}, s-r\right\},
$$

we have $0<r+\kappa<s$, and hence

$$
\begin{aligned}
r^{-1} \sup & \left\{d(f(b), f(a)) \mid a \in \mathbf{B}_{M}(b, r)\right\} \\
& \leq r^{-1} \sup \left\{d(f(b), f(a)) \mid a \in \mathbb{B}_{M}(b, r+\kappa)\right\} \\
& =r^{-1}(r+\kappa)(r+\kappa)^{-1} \sup \left\{d(f(b), f(a)) \mid a \in \mathbb{B}_{M}(b, r+\kappa)\right\} \\
& <r^{-1}(r+\kappa)\left(\alpha+2^{-1} \varepsilon\right) \\
& <\left(\alpha+2^{-1} \varepsilon\right)+\kappa r^{-1}\left(\alpha+2^{-1} \varepsilon\right) \\
& <\alpha+\frac{1}{2} \varepsilon+\frac{1}{2} \varepsilon \\
& =\alpha+\varepsilon .
\end{aligned}
$$

\footnotetext{
${ }^{3}$ The term calmness also occurs in the literature [6, Section 1.3].
} 
Since $r \in(0, s)$ was chosen arbitrarily, we obtain

$$
\sup _{r \in(0, s)}\left(r^{-1} \sup \left\{d(f(b), f(a)) \mid a \in \mathbf{B}_{M}(b, r)\right\}\right) \leq \alpha+\varepsilon .
$$

Since $\varepsilon>0$ was chosen arbitrarily, we obtain

$$
\limsup _{r \rightarrow 0^{+}}\left(r^{-1} \sup \left\{d(f(b), f(a)) \mid a \in \mathbf{B}_{M}(b, r)\right\}\right) \leq \alpha .
$$

Lemma 2.8. Let $Y$ and $X$ be normed spaces. Let $y \in \mathbf{S}_{Y}, \beta>0$, and let $\underline{\rho} \in$ $C\left(\mathbf{S}_{Y}, X\right)$ be pointwise $\beta$-Lipschitz at $y$. Then the positively homogeneous extension $\rho: Y \rightarrow X$ of $\underline{\rho}$, defined by

$$
\rho(z):=\left\{\begin{array}{ll}
0 & \text { if } z=0 \\
\|z\| \underline{\rho}\left(\frac{z}{\|z\|}\right) & \text { if } z \neq 0,
\end{array} \quad(z \in Y),\right.
$$

is continuous and is pointwise $\left(2 \beta+\|\underline{\rho}\|_{\infty}\right)$-Lipschitz on the set $\{\lambda y \in Y \mid \lambda>0\}$.

Proof. That $\rho$ is continuous is a straightforward exercise using reverse triangle inequality and the boundedness of $\rho$.

Let $\varepsilon>0$ be arbitrary. Since $\rho$ is pointwise $\beta$-Lipschitz at $y$, there exists some $R \in(0,1)$, such that, for all $r \in \overline{(0}, R)$,

$$
r^{-1} \sup \left\{\|\underline{\rho}(y)-\underline{\rho}(x)\| \mid x \in \mathbf{B}_{Y}(y, r) \cap \mathbf{S}_{Y}\right\}<\beta+2^{-1} \varepsilon .
$$

Let $z \in\{\lambda y \in Y \mid \lambda>0\}$ and $s \in\left(0,2^{-1}\|z\| R\right)$ be arbitrary. For any $x \in$ $\mathbf{B}_{Y}(z, s)$, we note that $x \neq 0$, since $s<\|z\|$. Furthermore,

$$
\begin{aligned}
\left\|\frac{z}{\|z\|}-\frac{x}{\|x\|}\right\| & \leq\left\|\frac{z}{\|z\|}-\frac{x}{\|z\|}\right\|+\left\|\frac{x}{\|z\|}-\frac{x}{\|x\|}\right\| \\
& =\frac{1}{\|z\|}\|z-x\|+\left|\frac{1}{\|z\|}-\frac{1}{\|x\|}\right|\|x\| \\
& \leq \frac{s}{\|z\|}+\mid \frac{\|x\|-\|z\|}{\|z\|\|x\|}\|x\| \\
& \leq \frac{s}{\|z\|}+\frac{1}{\|z\|}\|z-x\| \\
& \leq \frac{2 s}{\|z\|} \\
& <R .
\end{aligned}
$$

Therefore, for any $x \in \mathbf{B}_{Y}(z, s)$, we have

$$
\left\|y-\frac{x}{\|x\|}\right\|=\left\|\frac{z}{\|z\|}-\frac{x}{\|x\|}\right\| \leq \frac{2 s}{\|z\|}<R,
$$

and hence,

$$
\begin{aligned}
s^{-1}\|\rho(z)-\rho(x)\| & =s^{-1}\|\| z\left\|\underline{\rho}\left(\frac{z}{\|z\|}\right)-\right\| x\left\|\underline{\rho}\left(\frac{x}{\|x\|}\right)\right\| \\
\leq & s^{-1}\|\| z\left\|\underline{\rho}\left(\frac{z}{\|z\|}\right)-\right\| z\left\|\underline{\rho}\left(\frac{x}{\|x\|}\right)\right\| \\
& +s^{-1}\|\| z\left\|\underline{\rho}\left(\frac{x}{\|x\|}\right)-\right\| x\left\|\underline{\rho}\left(\frac{x}{\|x\|}\right)\right\| \\
= & s^{-1}\|z\|\left\|\underline{\rho}\left(\frac{z}{\|z\|}\right)-\underline{\rho}\left(\frac{x}{\|x\|}\right)\right\| \\
& +s^{-1} \mid\|z\|-\|x\|\left\|\underline{\rho}\left(\frac{x}{\|x\|}\right)\right\|
\end{aligned}
$$




$$
\begin{aligned}
& \leq s^{-1}\|z\|\left\|\underline{\rho}\left(\frac{z}{\|z\|}\right)-\underline{\rho}\left(\frac{x}{\|x\|}\right)\right\|+s^{-1}\|z-x\|\|\underline{\rho}\|_{\infty} \\
& \leq 2\left(\frac{2 s}{\|z\|}\right)^{-1}\left\|\underline{\rho}(y)-\underline{\rho}\left(\frac{x}{\|x\|}\right)\right\|+s^{-1} s\|\underline{\rho}\|_{\infty} \\
& <2\left(\beta+2^{-1} \varepsilon\right)+\|\underline{\rho}\|_{\infty} \\
& =2 \beta+\|\underline{\rho}\|_{\infty}+\varepsilon .
\end{aligned}
$$

Since $s \in\left(0,2^{-1}\|z\| R\right)$ was chosen arbitrarily, we obtain

$$
\sup _{s \in\left(0,2^{-1}\|z\| R\right)}\left(s^{-1}\left\{\|\rho(z)-\rho(x)\| \mid x \in \mathbf{B}_{Y}(z, s)\right\}\right) \leq 2 \beta+\|\underline{\rho}\|_{\infty}+\varepsilon .
$$

Since $\varepsilon>0$ was chosen arbitrarily, we have

$$
\limsup _{r \rightarrow 0^{+}}\left(r^{-1}\left\{\|\rho(z)-\rho(x)\| \mid x \in \mathbf{B}_{Y}(z, r)\right\}\right) \leq 2 \beta+\|\underline{\rho}\|_{\infty} .
$$

Finally, since $z$ was chosen arbitrarily from $\{\lambda y \in Y \mid \lambda>0\}$, we conclude that $\rho$ is pointwise $\left(2 \beta+\|\underline{\rho}\|_{\infty}\right)$-Lipschitz on $\{\lambda y \in Y \mid \lambda>0\}$.

\section{Main Result: A Pointwise Lipschitz Selection Theorem}

In this section we will prove our Pointwise Lipschitz Selection Theorem (Theorem 3.4).

For the sake of brevity and clarity of the proof, the results in this section is stated under the somewhat synthetic assumption of a correspondence admitting local strongly pointwise Lipschitz selections. Section 4 introduces a more natural property which we call lower pointwise Lipschitz-ness which allows for the statement of more natural versions of Theorem 3.4 .

Definition 3.1. Let $\alpha \geq 0$ and $(M, d)$ and $\left(M^{\prime}, d^{\prime}\right)$ be metric spaces and let $b \in M$. Let $\varphi: M \rightarrow M^{\prime}$ be a correspondence. We will say that $\varphi$ admits local strongly pointwise $\alpha$-Lipschitz selections at $b$ if, for every $y \in \varphi(b)$, there exists some open neighborhood $U \subseteq M$ of $b$ and a continuous selection $f: M \rightarrow M^{\prime}$ of $\varphi$ satisfying $f(b)=y$ with the restriction $\left.f\right|_{U}: U \rightarrow M^{\prime}$ strongly pointwise $\alpha$-Lipschitz at $b$ (as defined in Definition 2.6).

We will say $\varphi$ admits local strongly pointwise $\alpha$-Lipschitz selections, if it admits local strongly pointwise $\alpha$-Lipschitz selections at every point of $M$. If we may choose the neighborhood $U$ as the whole space $M$, we will omit the 'local' modifier, by saying $\varphi$ admits strongly pointwise $\alpha$-Lipschitz selections (at b).

With this definition in hand, we can turn toward establishing our Pointwise Lipschitz Selection Theorem (Theorem 3.4). The proof is somewhat delicate and is split into two parts. We briefly describe the argument employed:

The first and most technical part is given in the proof of Lemma 3.2. Given a non-empty- and convex-valued correspondence that admits local strongly pointwise Lipschitz selections, we start with any continuous selection $f_{0}$ of this correspondence. We inductively construct a sequence of selections $\left\{f_{n}\right\}$ of the correspondence in such a way that, for each $n \in \mathbb{N}$, the selection $f_{n}$ is pointwise Lipschitz at more points than its predecessor in the sequence $f_{n-1}$. This is achieved by making subtle adjustments to $f_{n-1}$. It is necessary to use a delicate hand in the construction of $f_{n}$ from $f_{n-1}$ to ensure that one does not disturb $f_{n-1}$ at the points where it is already pointwise Lipschitz. We do this by carefully adjusting a selection in the sequence only at points that form part of a sequence of successively finer maximal separations (denoted by $\left\{B_{n}\right\}$ in Lemma 3.2). This process yields precise control over the distance from the points where $f_{n-1}$ is already pointwise Lipschitz and points where it is safe to adjust $f_{n-1}$. We exploit this control together with a 
standard partition of unity argument and the assumption that the correspondence admits strongly pointwise Lipschitz selections to then carefully adjust $f_{n-1}$ to form its successor $f_{n}$.

The second part is given in the proof of Theorem 3.4. Using a sequence of selections of the correspondence $\left\{f_{n}\right\}$, as obtained from Lemma 3.2, it is easily seen that this sequence is uniform Cauchy and hence converges to a continuous selection of the correspondence. The bulk of the proof of Theorem 3.4 is a verification of the properties of the limit of this sequence, in particular that it is pointwise Lipschitz on a dense set of its domain.

Lemma 3.2. Let $(M, d)$ be a metric space and $X$ a normed space. Let $\alpha \geq 0$ and $\varphi: M \rightarrow X$ be a non-empty-and convex-valued correspondence that admits local strongly pointwise $\alpha$-Lipschitz selections (as defined in Definition [3.1). Then, for every $\varepsilon>0$ and any continuous selection $f_{0}$ of $\varphi$, there exists a sequence of continuous functions $\left\{f_{n}: M \rightarrow X\right\}$ and a sequence of subsets $\left\{B_{n}\right\}$ of $M$ such that, for every $n \in \mathbb{N}$ :

(1) The set $B_{n}$ is a maximal $2^{-(n-1)}$-separation in $M$ with $B_{n-1} \subseteq B_{n}$ (where we take $\left.B_{0}:=\emptyset\right)$.

(2) The function $f_{n}$ is a continuous selection of $\varphi$. If $f_{0}$ is bounded, then so is $f_{n}$.

(3) We have $\sup _{a \in M}\left\|f_{n}(a)-f_{n-1}(a)\right\| \leq 2^{-n} \varepsilon$.

(4) The function $f_{n}$ is pointwise $\alpha$-Lipschitz at every point of $B_{n}$.

(5) For every $b \in B_{n} \backslash B_{n-1}$ there exists a number $\delta_{b}^{(n)}>0$ so that, for every $a \in \mathbb{B}_{M}\left(b, \delta_{b}^{(n)}\right)$, we have $\left\|f_{n}(b)-f_{n}(a)\right\| \leq \alpha d(b, a)$.

(6) For any $k \in\{1, \ldots, n-1\}$ and $b \in B_{k}$, the function $f_{n}$ coincides with all the functions $f_{n-1}, \ldots, f_{k}$ on $\mathbb{B}_{M}\left(b, 2^{-n}\right)$, that is,

$$
\left.f_{n}\right|_{\mathbb{B}_{M}\left(b, 2^{-n}\right)}=\left.f_{n-1}\right|_{\mathbb{B}_{M}\left(b, 2^{-n}\right)}=\ldots=\left.f_{k}\right|_{\mathbb{B}_{M}\left(b, 2^{-n}\right)} .
$$

Proof. Let $\varepsilon>0$ be arbitrary. Let $B_{0}:=\emptyset$ and let $f_{0}: M \rightarrow X$ be a continuous selection of $\varphi$.

We proceed inductively. Let $n \in \mathbb{N}$ be arbitrary, and assume that the functions $f_{1}, \ldots f_{n-1}$ and the sets $B_{1}, \ldots, B_{n-1}$ have been defined to satisfy (1)-(6) in the statement of the result. We will construct $f_{n}: M \rightarrow X$ and $B_{n}$.

Firstly, by Lemma 2.4 there exists a maximal $2^{-(n-1)}$-separation in $M$, denoted by $B_{n}$, satisfying $B_{n-1} \subseteq B_{n}$.

For every $b \in B_{n} \backslash B_{n-1}$, by our assumption of $\varphi$ admitting local strongly pointwise $\alpha$-Lipschitz selections, there exists some $r_{b}>0$ and a continuous selection $g_{b}: M \rightarrow X$ of $\varphi$ satisfying $g_{b}(b)=f_{n-1}(b)$ with the restriction $\left.g_{b}\right|_{\mathbb{B}_{M}\left(b, r_{b}\right)}$ strongly pointwise $\alpha$-Lipschitz at $b$. In particular, $g_{b}$ is pointwise $\alpha$-Lipschitz at $b$. The map $M \ni a \mapsto\left\|f_{n-1}(a)-g_{b}(a)\right\|$ is continuous, hence there exists some $\delta_{b}^{(n)}>0$ with $\delta_{b}^{(n)}<\min \left\{2^{-(n+1)}, r_{b}\right\}$ such that, if $a \in \mathbb{B}_{M}\left(b, 2 \delta_{b}^{(n)}\right)$, then

$$
\left|\left\|f_{n-1}(a)-g_{b}(a)\right\|-\left\|f_{n-1}(b)-g_{b}(b)\right\|\right|<2^{-n} \varepsilon .
$$

But, since $f_{n-1}(b)=g_{b}(b)$, we have $\left\|f_{n-1}(a)-g_{b}(a)\right\|<2^{-n} \varepsilon$ for every $a \in$ $\mathbb{B}_{M}\left(b, 2 \delta_{b}^{(n)}\right)$.

We define the collections

$$
\mathcal{U}:=\left\{\mathbb{B}_{M}\left(b, 2 \delta_{b}^{(n)}\right) \mid b \in B_{n} \backslash B_{n-1}\right\} \text { and } \mathcal{C}:=\left\{\mathbf{B}_{M}\left(b, \delta_{b}^{(n)}\right) \mid b \in B_{n} \backslash B_{n-1}\right\} .
$$


Since $B_{n}$ is a $2^{-(n-1)}$-separation in $M$ and, for every $b \in B_{n} \backslash B_{n-1}$, we have $\delta_{b}^{(n)}<2^{-(n+1)}$, the elements of $\mathcal{U}$ are pairwise disjoint. Similarly, the elements of $\mathcal{C}$ are pairwise disjoint. Furthermore, it can be seen that $\cup \mathcal{C}$ is closed (see [7, III.9.2]).

We define $\mathcal{V}:=\mathcal{U} \cup\{M \backslash \cup \mathcal{C}\}$, which is an open cover of $M$. Since $M$ is paracompact (cf. Theorem 2.2), there exists a locally finite partition of unity $\left\{\rho_{V} \mid V \in \mathcal{V}\right\}$ subordinate to $\mathcal{V}[7$, Theorem 4.2, p.170]. For $V \in \mathcal{V}$, if $V=M \backslash \cup \mathcal{C}$, we define $h_{V}:=f_{n-1}$. If $V=\mathbb{B}_{M}\left(b, 2 \delta_{b}^{(n)}\right)$ for some $b \in B_{n} \backslash B_{n-1}$, we define $h_{V}:=g_{b}$. Finally, we define $f_{n}: M \rightarrow X$ as

$$
f_{n}(a):=\sum_{V \in \mathcal{V}} \rho_{V}(a) h_{V}(a) \quad(a \in M) .
$$

Since $\varphi$ is convex-valued, and, for every $V \in \mathcal{V}$, the function $h_{V}$ is a continuous selection of $\varphi$, we have that $f_{n}$ is a continuous selection of $\varphi$.

We claim that $\sup _{a \in M}\left\|f_{n-1}(a)-f_{n}(a)\right\| \leq 2^{-n} \varepsilon$. Let $a \in M$ be arbitrary. We distinguish two cases: Firstly, if $a \notin \cup \mathcal{U}$, then $a \in M \backslash \cup \mathcal{C}$ and therefore $\left\|f_{n-1}(a)-f_{n}(a)\right\|=0$. Secondly, if $a \in \mathcal{U}$, then, since the elements of $\mathcal{U}$ are disjoint, there exists a unique $b \in B_{n} \backslash B_{n-1}$ so that $a \in \mathbb{B}_{M}\left(b, 2 \delta_{b}^{(n)}\right)=: V$ and $h_{V}=g_{b}$. Then, with $W:=M \backslash \cup \mathcal{C}$, we have $h_{W}=f_{n-1}$. By definition of $\delta_{b}^{(n)}$, we see that

$$
\begin{aligned}
\left\|f_{n-1}(a)-f_{n}(a)\right\| & =\left\|f_{n-1}(a)-\sum_{U \in\{V, W\}} \rho_{U}(a) h_{U}(a)\right\| \\
& \leq\left|\rho_{V}(a)\right|\left\|f_{n-1}(a)-h_{V}(a)\right\| \\
& \quad+\left|\rho_{W}(a)\right|\left\|f_{n-1}(a)-h_{W}(a)\right\| \\
& \leq\left\|f_{n-1}(a)-g_{b}(a)\right\| \\
& <2^{-n} \varepsilon
\end{aligned}
$$

establishing our claim that $\sup _{a \in M}\left\|f_{n-1}(a)-f_{n}(a)\right\| \leq 2^{-n} \varepsilon$. If $f_{0}$ is bounded, then $f_{n-1}$ is bounded by assumption, and hence it is clear that $f_{n}$ is also bounded.

We notice, by construction, for every $b \in B_{n} \backslash B_{n-1}$ we have $\mathbb{B}_{M}\left(b, \delta_{b}^{(n)}\right) \cap(M \backslash \cup$ $\mathcal{C})=\emptyset$. Therefore $\left.f_{n}\right|_{\mathbb{B}_{M}\left(b, \delta_{b}^{(n)}\right)}=\left.g_{b}\right|_{\mathbb{B}_{M}\left(b, \delta_{b}^{(n)}\right)}$. Because $\delta_{b}^{(n)}<r_{b}$, the restriction $\left.g_{b}\right|_{\mathbb{B}_{M}\left(b, \delta_{b}^{(n)}\right)}$ is strongly pointwise $\alpha$-Lipschitz at $b$, and hence, the map $f_{n}$ is pointwise $\alpha$-Lipschitz at $b$.

Again by construction, for any $k \in\{1, \ldots, n-1\}$ and $b \in B_{k} \subseteq B_{n-1}$, we have $\mathbb{B}_{M}\left(b, 2^{-n}\right) \cap(\cup \mathcal{U})=\emptyset$ so that $\left.f_{n}\right|_{\mathbb{B}_{M}\left(b, 2^{-n}\right)}=\left.f_{n-1}\right|_{\mathbb{B}_{M}\left(b, 2^{-n}\right)}$. Since $f_{n-1}$ was assumed to be pointwise $\alpha$-Lipschitz at $b$, so is $f_{n}$. Furthermore, if $k<n-1$, by our initial assumption, we have

$$
\left.f_{n-1}\right|_{\mathbb{B}_{M}\left(b, 2^{-(n-1)}\right)}=\left.f_{n-2}\right|_{\mathbb{B}_{M}\left(b, 2^{-(n-1)}\right)}=\ldots=\left.f_{k}\right|_{\mathbb{B}_{M}\left(b, 2^{-(n-1)}\right)},
$$

and since $\mathbb{B}_{M}\left(b, 2^{-n}\right) \subseteq \mathbb{B}_{M}\left(b, 2^{-(n-1)}\right)$, we conclude

$$
\left.f_{n}\right|_{\mathbb{B}_{M}\left(b, 2^{-n}\right)}=\left.f_{n-1}\right|_{\mathbb{B}_{M}\left(b, 2^{-n}\right)}=\ldots=\left.f_{k}\right|_{\mathbb{B}_{M}\left(b, 2^{-n}\right)} .
$$

Remark 3.3. With $\left\{f_{n}\right\}$ and $\left\{B_{n}\right\}$ as constructed in the previous lemma, we note, for every $b \in \bigcup_{n \in \mathbb{N}} B_{n}$, the sequence $\left\{f_{n}(b)\right\} \subseteq X$ is eventually constant. Specifically, if for some $n \in \mathbb{N}$, we have $b \in B_{n}$, then $f_{m}(b)=f_{n}(b)$ for all $m \geq n$. We will use this fact in the proof of Theorem 3.4.

Theorem 3.4 (Pointwise Lipschitz Selection Theorem). Let $(M, d)$ be a metric space and $X$ a Banach space. Let $\alpha \geq 0$ and let $\varphi: M \rightarrow X$ be a non-empty-, closed-, and convex-valued correspondence that admits local strongly pointwise $\alpha-$ Lipschitz selections (as defined in Definition 3.1). If $\varphi$ has a (bounded) continuous 
selection, then, for every $\beta>\alpha$, there exists a (bounded) continuous selection of $\varphi$ that is pointwise $\beta$-Lipschitz (as defined in Definition [2.6) on a dense set of $M$.

Proof. Let $f_{0}: M \rightarrow X$ be a continuous selection of $\varphi$ and let $\beta>\alpha$ be arbitrary. Define $\varepsilon:=3^{-1}(\beta-\alpha)>0$, and with this $\varepsilon$ and $f_{0}$, let $\left\{f_{n}\right\}$ and $\left\{B_{n}\right\}$ be as obtained from Lemma 3.2. Since the family of sequences $\left\{f_{n}(a)\right\} \subseteq X$ are uniformly Cauchy with respect to $a \in M$, a standard exercise shows that the pointwise limit $f: M \rightarrow X$ defined by $f(a):=\lim _{n \rightarrow \infty} f_{n}(a)$ for all $a \in M$ is continuous. Since $\varphi$ is closed-valued, and each $f_{n}$ is a continuous selection of $\varphi$, the limit $f$ is also a continuous selection of $\varphi$. If $f_{0}$ is bounded, the sequence $\left\{f_{n}\right\}$ is Cauchy in the Banach space $C(M, X)$, and hence the limit $f$ is also bounded.

We let $B:=\bigcup_{n \in \mathbb{N}} B_{n}$, and by Lemma 2.5, the set $B$ is dense in $M$. We claim that $f$ is pointwise $\beta$-Lipschitz on $B$.

Let $b \in B$ be arbitrary. Let $N \in \mathbb{N}$ be the least number such that $b \in B_{N}$. With $\delta_{b}^{(N)}>0$ as obtained from Lemma 3.2, let $K \in \mathbb{N}$ be the least number satisfying $K \geq N$ and $2^{-K}<\delta_{b}^{(N)}$. Let $r \in\left(0,2^{-K}\right)$ be arbitrary, and let $n \in \mathbb{N}$ be such that $n \geq K$ and $r \in\left[2^{-(n+1)}, 2^{-n}\right)$. Let $y \in \mathbb{B}_{M}(b, r)$ be arbitrary. If $y=b$, then $r^{-1}\|f(b)-f(y)\|=0$. On the other hand, if $y \neq b$, since $\mathbb{B}_{M}(b, r)$ is open and $f$ is continuous, there exists some $\delta>0$ such that both $\mathbb{B}_{M}(y, \delta) \subseteq \mathbb{B}_{M}(b, r)$, and $\|f(y)-f(x)\|<r \varepsilon$ for all $x \in \mathbb{B}_{M}(y, \delta)$. By the density of $B$ in $M$, there exists some $c \in \mathbb{B}_{M}(y, \delta) \cap B$. Let $m \geq n$ be such that $c \in B_{m}$.

Now, by construction of the sequence $\left\{f_{n}\right\}$ in Lemma 3.2 (cf. Remark 3.3), we have $f(b)=f_{n}(b)$ and $f(c)=f_{m}(c)$, and

$$
\left.f_{n}\right|_{\mathbb{B}_{M}\left(b, 2^{-n}\right)}=\left.f_{n-1}\right|_{\mathbb{B}_{M}\left(b, 2^{-n}\right)}=\ldots=\left.f_{N}\right|_{\mathbb{B}_{M}\left(b, 2^{-n}\right)} .
$$

Furthermore, since

$$
c \in \mathbb{B}_{M}(y, \delta) \subseteq \mathbb{B}_{M}(b, r) \subseteq \mathbb{B}_{M}\left(b, 2^{-n}\right) \subseteq \mathbb{B}_{M}\left(b, \delta_{b}^{(N)}\right),
$$

again by Lemma 3.2, we have $\left\|f_{N}(b)-f_{N}(c)\right\| \leq \alpha d(b, c)<\alpha r$. Finally, keeping in mind that $\sup _{a \in M}\left\|f_{j}(a)-f_{j-1}(a)\right\|<2^{-j} \varepsilon$ for all $j \in \mathbb{N}$, and that $r \geq 2^{-(n+1)}$, we obtain

$$
\begin{aligned}
r^{-1} \| & f(b)-f(y) \| \\
& \leq r^{-1}\|f(b)-f(c)\|+r^{-1}\|f(c)-f(y)\| \\
& =r^{-1}\|f(b)-f(c)\|+r^{-1} r \varepsilon \\
& =r^{-1}\left\|f_{n}(b)-f_{m}(c)\right\|+\varepsilon \\
& \leq r^{-1}\left\|f_{n}(b)-f_{n}(c)\right\|+\left(r^{-1} \sum_{j=n+1}^{m}\left\|f_{j-1}(c)-f_{j}(c)\right\|\right)+\varepsilon \\
& \leq r^{-1}\left\|f_{n}(b)-f_{n}(c)\right\|+\left(r^{-1} \sum_{j=n+1}^{m} 2^{-j} \varepsilon\right)+\varepsilon \\
& <r^{-1}\left\|f_{n}(b)-f_{n}(c)\right\|+2^{n+1} 2^{-n} \varepsilon+\varepsilon \\
& =r^{-1}\left\|f_{N}(b)-f_{N}(c)\right\|+3 \varepsilon \\
& \leq r^{-1} \alpha d(b, c)+3 \varepsilon \\
& <r^{-1} r \alpha+3 \varepsilon \\
& =\alpha+\beta-\alpha . \\
& =\beta .
\end{aligned}
$$

Since $y \in \mathbb{B}_{M}(b, r)$ was chosen arbitrarily, we have

$$
r^{-1} \sup \left\{\|f(b)-f(y)\| \mid y \in \mathbb{B}_{M}(b, r)\right\} \leq \beta .
$$


But $r \in\left(0,2^{-K}\right)$ was also chosen arbitrarily, and therefore

$$
\limsup _{r \rightarrow 0^{+}}\left(r^{-1} \sup \left\{\|f(b)-f(y)\| \mid y \in \mathbb{B}_{M}(b, r)\right\}\right) \leq \beta .
$$

By Lemma 2.7 the function $f$ is pointwise $\beta$-Lipschitz at $b \in B$. Finally, since $b \in B$ was chosen arbitrarily, we conclude that $f$ is pointwise $\beta$-Lipschitz on $B$ which is dense in $M$.

Remark 3.5. We point out that a function that is pointwise $\alpha$-Lipschitz for some $\alpha>0$ on a dense set of its domain is not necessarily pointwise $\beta$-Lipschitz for some $\beta>0$ on the whole of its domain. The Cantor function [15, Exercise 1.6.48], which maps $[0,1]$ to $[0,1]$, is pointwise 0 -Lipschitz on the complement of the Cantor set (which is dense in $[0,1]$ ), while it is not pointwise $\alpha$-Lipschitz for any $\alpha>0$ on the whole interval. An easy way to see this is to apply Theorem 6.3. noting that the Cantor function is not Lipschitz, while $[0,1]$ is a length space (cf. 4, Definition 2.1.6]).

\section{LOWER POINTWISE LIPSCHITZ CORRESPONDENCES}

The condition of a correspondence admitting local strongly pointwise Lipschitz selections in the hypothesis Theorem 3.4 is admittedly somewhat synthetic. In this section we will show that a more natural condition, which we call "lower pointwise Lipschitz-ness" of a correspondence, is a sufficient condition for a correspondence to admit local strongly pointwise Lipschitz selections.

Definition 4.1. Let $(M, d)$ be a metric space, $X$ a normed space, and $\alpha \geq 0$. A correspondence $\varphi: M \rightarrow X$ will be said to be lower pointwise $\alpha$-Lipschitz at $b \in M$, if, for every $y \in \varphi(b)$ and $a \in M$, the set

$$
\varphi(a) \cap\left(y+\alpha d(b, a) \mathbf{B}_{X}\right)
$$

is non-empty. We will say that $\varphi$ is lower pointwise $\alpha$-Lipschitz if it is lower pointwise $\alpha$-Lipschitz at every point of $M$.

The following few results are fairly straightforward in nature, if somewhat technical. Our aim is to prove Proposition 4.4 which gives sufficient conditions for a correspondence to admit strongly pointwise Lipschitz selections. The bulk of the work is done in Proposition 4.3 which establishes the lower hemicontinuity of a certain correspondence derived from one that is assumed to be lower pointwise Lipschitz. A straightforward application of Michael's Selection Theorem will then establish Proposition 4.4 .

Lemma 4.2. Let $X$ be a normed space. Let $\alpha \geq 0, x \in X$ and $G \subseteq X$ be a convex set such that, for every $\varepsilon>0$, the set $G \cap\left(x+(\alpha+\varepsilon) \mathbf{B}_{X}\right)$ is non-empty. If, for an open set $U \subseteq X$ and some $\varepsilon_{0}>0$, the set $G \cap\left(x+\left(\alpha+\varepsilon_{0}\right) \mathbf{B}_{X}\right) \cap U$ is non-empty, then $G \cap\left(x+\left(\alpha+\varepsilon_{0}\right) \mathbb{B}_{X}\right) \cap U$ is also non-empty.

Proof. Let $U \subseteq X$ be open and $\varepsilon_{0}>0$ such that $G \cap\left(x+\left(\alpha+\varepsilon_{0}\right) \mathbf{B}_{X}\right) \cap U \neq \emptyset$. Let $y \in G \cap\left(x+\left(\alpha+\varepsilon_{0}\right) \mathbf{B}_{X}\right) \cap U$. If $y \in x+\left(\alpha+\varepsilon_{0}\right) \mathbb{B}_{X}$, then we are done. We therefore assume that $y \in x+\left(\alpha+\varepsilon_{0}\right) \mathbf{S}_{X}$. Let $z \in G \cap\left(x+\left(\alpha+2^{-1} \varepsilon_{0}\right) \mathbf{B}_{X}\right) \neq \emptyset$. Then, for every $t \in(0,1]$,

$$
\begin{aligned}
\|t z+(1-t) y-x\| & =\|t z+(1-t) y-t x-(1-t) x\| \\
& \leq t\|z-x\|+(1-t)\|y-x\| \\
& \leq t\left(\alpha+2^{-1} \varepsilon_{0}\right)+(1-t)\left(\alpha+\varepsilon_{0}\right) \\
& <t\left(\alpha+\varepsilon_{0}\right)+(1-t)\left(\alpha+\varepsilon_{0}\right) \\
& =\left(\alpha+\varepsilon_{0}\right) .
\end{aligned}
$$


In other words, $t z+(1-t) y \in x+\left(\alpha+\varepsilon_{0}\right) \mathbb{B}_{X}$ for all $t \in(0,1]$. Since $[0,1] \ni t \mapsto$ $t z+(1-t) y$ is continuous, there exists some $t_{0} \in(0,1]$ such that $t_{0} z+\left(1-t_{0}\right) y \in U$. Since $G$ is convex, $t_{0} z+\left(1-t_{0}\right) y \in G$. We conclude $t_{0} z+\left(1-t_{0}\right) y \in G \cap(x+(\alpha+$ $\left.\left.\varepsilon_{0}\right) \mathbb{B}_{X}\right) \cap U$.

Proposition 4.3. Let $(M, d)$ be a metric space, $X$ a normed space and $\alpha \geq 0$. Let $a \in M$ and let $\varphi: M \rightarrow X$ be a convex-valued lower hemicontinuous correspondence that is lower pointwise $(\alpha+\varepsilon)$-Lipschitz at $a \in M$ for every $\varepsilon>0$. Then, for every $y \in \varphi(a)$ and $\varepsilon>0$, the correspondence $\psi: M \rightarrow X$, defined by

$$
\psi(b):=\varphi(b) \cap\left(y+(\alpha+\varepsilon) d(a, b) \mathbf{B}_{X}\right) \quad(b \in M),
$$

is lower hemicontinuous. Moreover, $\psi$ is convex-and non-empty-valued.

Proof. Let $y \in \varphi(a)$ and $\varepsilon>0$ be arbitrary and let $\psi: M \rightarrow X$ be as defined in the statement of the result. That $\psi$ is convex-valued is immediate. That $\psi$ is non-empty-valued, follows from $\varphi$ being lower pointwise $(\alpha+\varepsilon)$-Lipschitz at $a \in M$.

We first show that $\psi$ is lower hemicontinuous at $a$. Let $U \subseteq X$ be an open set satisfying $\psi(a) \cap U \neq \emptyset$. Since $\psi(a)=\{y\}$, we have $y \in U$. Let $r>0$ be such that $y+r \mathbb{B}_{X} \subseteq U$. Since $\varphi$ is lower hemicontinuous, there exists some neighborhood $V \subseteq M$ of $a$ so that $b \in V$ implies that $\varphi(b) \cap\left(y+r \mathbb{B}_{X}\right) \neq \emptyset$. Let $0<s<(\alpha+\varepsilon)^{-1} r$ be such that $\mathbb{B}_{M}(a, s) \subseteq V$. Fix any $b \in \mathbb{B}_{M}(a, s)$. Since $\varphi$ is lower pointwise $(\alpha+\varepsilon)$ Lipschitz at $a \in M$ for every $\varepsilon>0$, the set $\psi(b)$ is non-empty, and hence there exists some $z \in \psi(b)$. But $z \in\left(y+(\alpha+\varepsilon) d(a, b) \mathbf{B}_{X}\right) \subseteq y+r \mathbb{B}_{X} \subseteq U$. Therefore $\psi(b) \cap U \neq \emptyset$ for all $b \in \mathbb{B}_{M}(a, s)$. We conclude that $\psi$ is lower hemicontinuous at a.

Let $c \in M \backslash\{a\}$ be arbitrary. We claim that $\psi$ is lower hemicontinuous at $c$. Let $U \subseteq X$ be an open set satisfying $\psi(c) \cap U \neq \emptyset$. Since $\varphi$ is lower pointwise $(\alpha+\varepsilon)$-Lipschitz at $a \in M$ for every $\varepsilon>0$, by Lemma 4.2, the set $\varphi(c) \cap$ $\left(y+(\alpha+\varepsilon) d(a, c) \mathbb{B}_{X}\right) \cap U$ is non-empty. Let

$$
z \in \varphi(c) \cap\left(y+(\alpha+\varepsilon) d(a, c) \mathbb{B}_{X}\right) \cap U,
$$

and choose $r>0$ satisfying both $0<r<(\alpha+\varepsilon) d(a, c)-\|y-z\|$ and

$$
z+r \mathbb{B}_{X} \subseteq\left(y+(\alpha+\varepsilon) d(a, c) \mathbb{B}_{X}\right) \cap U .
$$

Since $\varphi$ is lower hemicontinuous, there exists some neighborhood $V \subseteq M$ of $c$, such that $b \in V$ implies $\varphi(b) \cap\left(z+r \mathbb{B}_{X}\right) \neq \emptyset$. By definition of $r$, we have $(\alpha+\varepsilon) d(a, c)-\|y-z\|-r>0$, hence we choose $s>0$ to satisfy both

$$
0<s<d(a, c)-(\alpha+\varepsilon)^{-1}\|z-y\|-(\alpha+\varepsilon)^{-1} r
$$

and $\mathbb{B}_{M}(c, s) \subseteq V$. Then, for $b \in \mathbb{B}_{M}(c, s)$, by the reverse triangle inequality,

$$
\begin{aligned}
(\alpha+\varepsilon) d(a, b) & \geq(\alpha+\varepsilon) d(a, c)-(\alpha+\varepsilon) d(b, c) \\
& >(\alpha+\varepsilon) d(a, c)-(\alpha+\varepsilon) s \\
& >(\alpha+\varepsilon) d(a, c)-(\alpha+\varepsilon) d(a, c)+\|z-y\|+r \\
& =\|z-y\|+r .
\end{aligned}
$$

Hence, for $b \in \mathbb{B}_{M}(c, s)$ and any $w \in z+r \mathbb{B}_{X}$,

$$
\begin{aligned}
\|w-y\| & \leq\|w-z\|+\|z-y\| \\
& <r+\|z-y\| \\
& <(\alpha+\varepsilon) d(a, b),
\end{aligned}
$$

so that $\left(z+r \mathbb{B}_{X}\right) \subseteq\left(y+(\alpha+\varepsilon) d(a, b) \mathbb{B}_{X}\right)$ for all $b \in \mathbb{B}_{M}(c, s)$. By definition of $V$, for any $b \in \mathbb{B}_{M}(c, s) \subseteq V$, there exists some $z^{\prime} \in\left(z+r \mathbb{B}_{X}\right) \cap \varphi(b) \neq \emptyset$. But then $z^{\prime} \in\left(z+r \mathbb{B}_{X}\right) \subseteq U$ and

$$
z^{\prime} \in\left(z+r \mathbb{B}_{X}\right) \subseteq\left(y+(\alpha+\varepsilon) d(a, b) \mathbb{B}_{X}\right) \subseteq\left(y+(\alpha+\varepsilon) d(a, b) \mathbf{B}_{X}\right),
$$


so that $z^{\prime} \in \psi(b) \cap U$. Hence $\psi(b) \cap U \neq \emptyset$ for every $b \in \mathbb{B}_{M}(c, s)$, and therefore $\psi$ is lower hemicontinuous at $c$.

We finally conclude that $\psi$ is lower hemicontinuous.

Proposition 4.4. Let $(M, d)$ be a metric space, $X$ a Banach space and $\alpha \geq 0$. Let $\varphi: M \rightarrow X$ be a closed-and convex-valued lower hemicontinuous correspondence that is lower pointwise $(\alpha+\varepsilon)$-Lipschitz for every $\varepsilon>0$. Then, for every $\varepsilon>0$, the correspondence $\varphi$ admits strongly pointwise $(\alpha+\varepsilon)$-Lipschitz selections.

Proof. Let $\varepsilon>0, b \in M$ and $y \in \varphi(b)$ be arbitrary. By Proposition 4.3, the correspondence $\psi: M \rightarrow X$, defined by

$$
\psi(a):=\varphi(a) \cap\left(y+(\alpha+\varepsilon) d(b, a) \mathbf{B}_{X}\right) \quad(a \in M),
$$

is lower hemicontinuous, as well as being closed-, convex-, and non-empty-valued. Since $M$ is paracompact by Stone's Theorem (Theorem[2.2), by applying Michael's Selection Theorem (Theorem 2.1), there exists a continuous selection $f: M \rightarrow$ $X$ of $\psi$. By definition of $\psi$, the function $f$ is also a continuous selection of $\varphi$. Furthermore, $f$ is strongly pointwise $(\alpha+\varepsilon)$-Lipschitz at $b \in M$ and satisfies $f(b)=$ $y$.

Since $\varepsilon>0, b \in M$ and $y \in \varphi(b)$ were chosen arbitrarily, we conclude that $\varphi$ admits strongly pointwise $(\alpha+\varepsilon)$-Lipschitz selections for every $\varepsilon>0$.

Easy applications of Proposition 4.4 and our Pointwise Lipschitz Selection Theorem (Theorem 3.4) yield the following two corollaries. Compared to Theorem 3.4 these two corollaries give more natural (but less general) sufficient conditions on a correspondence for the existence of a continuous selection that is pointwise Lipschitz on a dense set of its domain.

Corollary 4.5. Let $(M, d)$ be a metric space, $X$ a Banach space and $\alpha \geq 0$. Let $\varphi: M \rightarrow X$ be a closed-and convex-valued lower hemicontinuous correspondence that is lower pointwise $(\alpha+\varepsilon)$-Lipschitz for every $\varepsilon>0$. Then, for any $\beta>\alpha$, there exists a continuous selection of $\varphi$ that is pointwise $\beta$-Lipschitz on a dense set of $M$.

If, additionally, there exists a bounded continuous selection of $\varphi$, then, for any $\beta>\alpha$, there also exists a bounded continuous selection of $\varphi$ that is pointwise $\beta$ Lipschitz on a dense set of $M$.

Proof. Since $\varphi$ is lower pointwise $(\alpha+\varepsilon)$-Lipschitz, it is also non-empty valued. By Michael's Selection Theorem (Theorem 2.1) $\varphi$ has a continuous selection. Let $\beta>\alpha$ and define $\varepsilon:=2^{-1}(\beta-\alpha)$. By Proposition 4.4, the correspondence $\varphi$ admits strongly pointwise $(\alpha+\varepsilon)$-Lipschitz selections. We note that $\alpha+\varepsilon<\alpha+2 \varepsilon=\beta$. Then, by Theorem 3.4 there exists a continuous selection of $\varphi$ that is pointwise $\beta$-Lipschitz on a dense set of $M$. Furthermore, if $\varphi$ has a bounded continuous selection, Theorem 3.4 ensures the existence of a bounded continuous selection of $\varphi$ that is pointwise $\beta$-Lipschitz on a dense set of $M$.

Corollary 4.6. Let $(M, d)$ be a bounded metric space, $X$ a Banach space and $\alpha \geq 0$. Let $\varphi: M \rightarrow X$ be a closed-and convex-valued lower hemicontinuous correspondence that is lower pointwise $(\alpha+\varepsilon)$-Lipschitz for every $\varepsilon>0$. Then, for any $\beta>\alpha$, there exists a bounded continuous selection of $\varphi$ that is pointwise $\beta$-Lipschitz on a dense set of $M$.

Proof. Let $\varepsilon>0$ be arbitrary. By Proposition 4.4, the correspondence $\varphi$ admits strongly pointwise $(\alpha+\varepsilon)$-Lipschitz selections. I.e., for any $b \in M$ and $y \in \varphi(b)$, there exists a continuous selection $f$ of $\varphi$ that is strongly pointwise $(\alpha+\varepsilon)$-Lipschitz selection at $b$. Since $M$ is bounded, this selection $f$ is a bounded continuous selection of $\varphi$. Applying Corollary 4.5 yields the result. 


\section{Application: An improved Bartle-Graves Theorem}

We recall the following version of the classical Bartle-Graves Theorem:

Theorem 5.1 (Classical Bartle-Graves Theorem [2, Corollary 17.67]). Let X and $Y$ be Banach spaces. Every continuous linear surjection $T: X \rightarrow Y$ has a continuous (and positively homogeneous) right inverse $\tau: Y \rightarrow X$ (i.e., $T \circ \tau=i d_{Y}$ ).

In this section we leverage our Pointwise Lipschitz Selection Theorem (Theorem 3.4) to establish a slight improvement of the classical Bartle-Graves Theorem. Since the case where $Y$ is finite dimensional is trivial (because the kernel of $T$ is then complemented), we restrict ourselves to the infinite dimensional case. In Theorem 5.2 we show that the map $\tau$ in the above theorem can, in fact, be chosen to be pointwise Lipschitz on a dense set of $Y$. The construction of this dense set, through application of Theorem 3.4, yields a meager set.

The proof of Theorem 5.2 is essentially a straightforward verification of the lower pointwise Lipschitz-ness of the inverse image correspondence (restricted to the unit sphere of the codomain). This allows us to apply Corollary 4.6 to complete the proof.

Theorem 5.2 (Improved Bartle-Graves Theorem). Let $X$ and $Y$ be infinite dimensional Banach spaces and $T: X \rightarrow Y$ a continuous linear surjection. Then there exist a constant $\eta>0$ and a positively homogeneous continuous right inverse $\tau: Y \rightarrow X$ of $T$ that is pointwise $\eta$-Lipschitz on a dense meager set of $Y$.

Proof. By the Open Mapping Theorem, there exists some $\gamma>0$ such that $\gamma \mathbb{B}_{Y} \subseteq$ $T\left(\mathbb{B}_{X}\right)$. We define the correspondence $\varphi: \mathbf{S}_{Y} \rightarrow X$ by $\varphi(y):=T^{-1}\{y\}$ for $y \in \mathbf{S}_{Y}$. It is clear that $\varphi$ is non-empty-, closed-, and convex-valued.

We claim that $\varphi$ is lower hemicontinuous. Let $y \in \mathbf{S}_{Y}$ be arbitrary and $U \subseteq X$ an open set satisfying $\varphi(y) \cap U \neq \emptyset$. Let $x \in \varphi(y) \cap U$ be arbitrary and let $r>0$ be such that $x+r \mathbb{B}_{X} \subseteq U$. Let $z \in \mathbf{S}_{Y} \cap\left(y+r \gamma \mathbb{B}_{Y}\right)$ be arbitrary. Define $z^{\prime}:=z-y$ so that $z^{\prime} \in r \gamma \mathbb{B}_{Y}$. Then there exists some $x^{\prime} \in r \mathbb{B}_{X}$ such that $T x^{\prime}=z^{\prime}$, and hence $T\left(x^{\prime}+x\right)=z^{\prime}+y=z-y+y=z$. Therefore $x+x^{\prime} \in \varphi(z) \cap\left(x+r \mathbb{B}_{X}\right)$, so that, for any $z \in \mathbf{S}_{Y} \cap\left(y+r \gamma \mathbb{B}_{Y}\right)$, we have $\varphi(z) \cap U \neq \emptyset$. We conclude that $\varphi$ is lower hemicontinuous.

Let $\varepsilon>0$ be arbitrary and set $\alpha:=\gamma^{-1}$. We claim that $\varphi$ is lower pointwise $(\alpha+\varepsilon)$-Lipschitz. Let $y \in \mathbf{S}_{Y}$ and $x \in \varphi(y)$ be arbitrary. For any $z \in \mathbf{S}_{Y}$, define $z^{\prime}:=z-y$. Then $z^{\prime} \in(1+\varepsilon \gamma)\|z-y\| \mathbb{B}_{Y}$. Let $x^{\prime} \in \gamma^{-1}(1+\varepsilon \gamma)\|z-y\| \mathbb{B}_{X}$ be such that $T x^{\prime}=z^{\prime}$. Then $T\left(x+x^{\prime}\right)=y+z^{\prime}=y+z-y=z$, so that $x+x^{\prime} \in \varphi(z)$. But $x^{\prime} \in$ $(\alpha+\varepsilon)\|z-y\| \mathbb{B}_{X} \subseteq(\alpha+\varepsilon)\|z-y\| \mathbf{B}_{X}$. Hence $\varphi(z) \cap\left(x+(\alpha+\varepsilon)\|z-y\| \mathbf{B}_{X}\right) \neq \emptyset$ and we conclude that $\varphi$ is lower pointwise $(\alpha+\varepsilon)$-Lipschitz for every $\varepsilon>0$.

Let $\beta>\alpha$. By Corollary 4.6, there exists a selection $\tau \in C\left(\mathbf{S}_{Y}, X\right)$ of the correspondence $\varphi$ that is pointwise $\beta$-Lipschitz on a dense set of $\mathbf{S}_{Y}$. We denote this dense set by $B \subseteq \mathbf{S}_{Y}$, which, by construction is meager (see the proof of Theorem 3.4 where $B$ is constructed as $\bigcup_{n \in \mathbb{N}} B_{n}$, with $B_{n}$ being a $2^{-(n-1)}$-separation for each $n \in \mathbb{N}$. Since $\mathbf{S}_{Y}$ was assumed to not be discrete, the set $B_{n}$ is nowhere dense in $\mathbf{S}_{Y}$ for each $n \in \mathbb{N}$ ).

It is straightforward to see that $B^{\prime}:=\{\lambda b \mid \lambda>0, b \in B\}$ is dense and meager in $Y$. By Lemma 2.8, the positively homogeneous extension $\tau: Y \rightarrow X$ of $\underline{\tau}$ is pointwise $\left(2 \beta+\|\underline{\tau}\|_{\infty}\right)$-Lipschitz on $B^{\prime}$. Setting $\eta:=2 \beta+\|\underline{\tau}\|_{\infty}$ and noting that $\tau$ is a right inverse of $T$ completes the proof. 


\section{An example of Aharoni and Lindenstrauss}

The following example, devised by Aharoni and Lindenstrauss [1], shows that continuous linear surjections between Banach spaces need not have Lipschitz or even uniformly continuous right inverses in general.

Example 6.1. Let $D$ denote the space of all bounded real-valued functions on $[0,1]$ that are right continuous at every point of $[0,1]$, whose left limit exists at every point of $[0,1]$, and with discontinuities only occurring at rational numbers. We endow $D$ with the uniform norm $\|\cdot\|_{\infty}$. Let $C([0,1]) \subseteq D$ denote the closed subspace of all continuous real-valued functions on $[0,1]$. The quotient map $q: D \rightarrow D / C([0,1])$ has no Lipschitz (even uniformly continuous) right inverse. We refer the reader to [1] or [3, Example 1.20] for proof of this fact.

Our improved Bartle-Graves Theorem (Theorem 5.2) shows that the quotient map $q$ has a continuous positively homogeneous right inverse that is pointwise $\eta$ Lipschitz for some $\eta>0$ on a dense meager set of $D / C([0,1])$. That the quotient map $q$ does not admit a Lipschitz right inverse shows that our Pointwise Lipschitz Selection Theorem (Theorem 3.4) cannot be improved to a general result which may yield a selection that is pointwise Lipschitz on the whole of its domain:

We first quote the following result by Schäffer [13, Theorem 3.6]:

Theorem 6.2. The unit sphere of every normed space is bi-Lipschitz homeomorphic to a length space (cf. 4, Definition 2.1.6]).

Next, a straightforward adaptation of a result due to Durand-Cartagena and Jaramillo [8, Corollary 2.4] yields the following result:

Theorem 6.3. Let $X$ be a normed space. If a metric space $(M, d)$ is bi-Lipschitz homeomorphic to a length space, then every function $f: M \rightarrow X$ that is pointwise $\alpha$-Lipschitz for some $\alpha \geq 0$ on the whole of $M$ is, in fact, Lipschitz.

Returning to Example 6.1, with the correspondence $\varphi: \mathbf{S}_{D / C([0,1])} \rightarrow D$ defined by $\varphi(x):=q^{-1}(\{x\})$ for all $x \in \mathbf{S}_{D / C([0,1])}$, should there exist a selection of $\varphi$ that is pointwise $\alpha$-Lipschitz on the whole of $\mathbf{S}_{D / C([0,1])}$, we could be able to conclude that such a selection is Lipschitz by Theorems 6.2 and 6.3. The positively homogeneous extension $f$ would then be a Lipschitz right inverse of $q$, contradicting Aharoni and Lindenstrauss' observation that no such map exists.

Acknowledgement. The author would like to thank the MathOverflow community (Nate Eldredge in particular, for pointing out the example in Remark 3.5 to the author), and the anonymous referees of the paper for their constructive comments and suggestions.

\section{REFERENCES}

1. I. Aharoni and J. Lindenstrauss, Uniform equivalence between Banach spaces, Bull. Amer. Math. Soc. 84 (1978), no. 2, 281-283.

2. C.D. Aliprantis and K.C. Border, Infinite dimensional analysis, third ed., Springer, Berlin, 2006.

3. Y. Benyamini and J. Lindenstrauss, Geometric nonlinear functional analysis. Vol. 1, American Mathematical Society, Providence, RI, 2000.

4. D. Burago, Y. Burago, and S. Ivanov, A course in metric geometry, American Mathematical Society, Providence, RI, 2001.

5. M. de Jeu and M. Messerschmidt, A strong open mapping theorem for surjections from cones onto Banach spaces, Adv. Math. 259 (2014), 43-66.

6. A.L. Dontchev and R.T. Rockafellar, Implicit functions and solution mappings, second ed., Springer, New York, 2014.

7. J. Dugundji, Topology, Allyn and Bacon, Inc., Boston, Mass.-London-Sydney, 1978. 
8. E. Durand-Cartagena and J.A. Jaramillo, Pointwise Lipschitz functions on metric spaces, J. Math. Anal. Appl. 363 (2010), no. 2, 525-548.

9. G. Godefroy and N.J. Kalton, Lipschitz-free Banach spaces, Studia Math. 159 (2003), no. 1, $121-141$.

10. N.J. Kalton, The nonlinear geometry of Banach spaces, Rev. Mat. Complut. 21 (2008), no. 1, 7-60.

11. M. Messerschmidt and M. Wortel, The intrinsic metric on the unit sphere of a normed space, arXiv:1510.07442.

12. K. Przesławski and D. Yost, Continuity properties of selectors and Michael's theorem, Michigan Math. J. 36 (1989), no. 1, 113-134.

13. J.J. Schäffer, Inner diameter, perimeter, and girth of spheres, Math. Ann. 173 (1967), 59-79; addendum, ibid. 173 (1967), 79-82.

14. A.H. Stone, Paracompactness and product spaces, Bull. Amer. Math. Soc. 54 (1948), 977-982.

15. T. Tao, An introduction to measure theory, vol. 126, American Mathematical Society, Providence, RI, 2011.

Miek Messerschmidt; Department of Mathematics and Applied Mathematics; University of Pretoria; Private bag X20 Hatfield; 0028 Pretoria; South Africa

E-mail address: mmesserschmidt@gmail.com 